\title{
A NOTE ON SPANNING TREES WITH A SPECIFIED DEGREE SEQUENCE
}

\author{
María Elena Martínez-Cuero \\ Departamento de Matemáticas \\ Universidad Autónoma Metropolitana-Iztapalapa \\ sherlyroses@hotmail.com \\ Eduardo Rivera-Campo \\ Departamento de Matemáticas \\ Universidad Autónoma Metropolitana-Iztapalapa \\ erc@xanum.uam.mx
}

\begin{abstract}
We give an Ore-Type condition sufficient for a graph $G$ to have a spanning tree with a specified degree sequence.
\end{abstract}

Keywords: Spanning Tree, Degree sequence, Arboreal.

\section{INTRODUCTION}

O. Ore [3] proved that if $G$ is a graph with $n$ vertices such that $d(u)+d(v) \geq n-1$ for each pair $u, v$ of non-adjacent vertices, then $G$ contains a hamiltonian path. This result has been generalized in many directions.

S. Win [5] showed that if $r \geq 2$ is an integer and $G$ is a connected graph with $n$ vertices such that $d\left(u_{1}\right)+d\left(u_{2}\right)+\cdots+d\left(u_{r}\right) \geq n-1$ for each set of $r$ independent vertices of $G$, then $G$ has a spanning tree with maximum degree at most $r$.

Years later, H. Broersma and H. Tuinstra [2] showed that if $s \geq 2$ is an integer and $G$ is a connected graph with $n$ vertices such that $d(u)+d(v) \geq n-s+1$ for each pair $u, v$ of non-adjacent vertices, then $G$ contains a spanning tree with at most $s$ vertices with degree 1 .

E. Rivera-Campo [4] gave a condition on the graph $G$ that bounds the degree of each vertex in a certain spanning tree $T$ of $G$ and the number of vertices of $T$ with degree 1 .

Theorem 1. Let $n, k$, and $d_{1}, d_{2}, \ldots, d_{n}$ be integers with $1 \leq k \leq n-1$ and $2 \leq d_{1} \leq d_{2} \leq$ $\cdots \leq d_{n} \leq n-1$. If $G$ is a $k$-connected graph with vertex set $V(G)=\left\{w_{1}, w_{2}, \ldots, w_{n}\right\}$ such

Partially supported by Conacyt, México. 
that $d(u)+d(v) \geq n-1-\sum_{j=1}^{n}\left(d_{j}-2\right)$ for each pair $u, v$ of non-adjacent vertices, then $G$ contains a spanning tree $T$ with at most $2+\sum_{j=1}^{n}\left(d_{j}-2\right)$ vertices with degree 1 and such that $d_{T}\left(w_{j}\right) \leq d_{j}$ for $j=1,2, \ldots, n$.

Let $n$ be a positive integer. An arboreal sequence is a sequence of positive integers $d_{1}, d_{2}, \ldots, d_{n}$ such that $\sum_{j=1}^{n} d_{j}=2(n-1)$. It is well known that a sequence $\sigma=d_{1}, d_{2}, \ldots, d_{n}$ is arboreal if and only if there is a tree whose vertices have degrees $d_{1}, d_{2}, \ldots, d_{n}$.

Let $\sigma=d_{1}, d_{2}, \ldots, d_{n}$ be an arboreal sequence and $G$ be a labelled graph with vertex set $V(G)=\left\{w_{1}, w_{2}, \ldots, w_{n}\right\}$. A spanning tree $T$ of $G$ has degree sequence $\sigma$ if $d_{T}\left(w_{i}\right)=d_{i}$ for $i=1,2, \ldots, n$. In this note we prove the following result:

Theorem 2. Let $n \geq 4$ be an integer and $G$ be a labelled graph with vertex set $V(G)=$ $\left\{w_{1}, w_{2}, \ldots, w_{n}\right\}$. If $d(u)+d(v) \geq \frac{3 n-1}{2}$ for each pair $u, v$ of non-adjacent vertices, then $G$ contains a spanning tree $T$ with degree sequence $\sigma$ for each arboreal sequence $\sigma=d_{1}, d_{2}, \ldots, d_{n}$ with $1 \leq d_{i} \leq 3$ for $i=1,2, \ldots, n$.

For each positive integer $k$ let $X_{k}=\left\{x_{1}, x_{2}, \ldots, x_{k}\right\}, Y_{k}=\left\{y_{1}, y_{2}, \ldots, y_{k}\right\}$ and $Z_{k}=$ $\left\{z_{1}, z_{2}, \ldots, z_{2 k+2}\right\}$ be pairwise disjoint sets of vertices and let $G_{k}$ be the complete graph with vertex set $X_{k} \cup Y_{k} \cup Z_{k}$ with all edges $x_{i} y_{i}, 1 \leq i, j \leq k$ removed. See Fig. 1 for the case $k=2$.

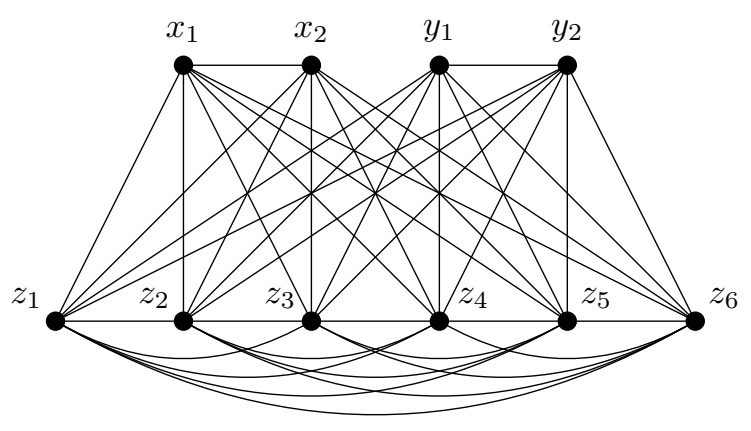

FIGURE 1. Graph $G_{2}$

We claim that the graph $G_{k}$ contains no spanning tree $T$ such that $d_{T}\left(x_{i}\right)=d_{T}\left(y_{i}\right)=3$ for $i=1,2, \ldots, k$ and $d_{T}\left(z_{j}\right)=1$ for $j=1,2, \ldots, 2 k+2$; for if $T$ is such a tree, then $T-Z_{k}$ would be a spanning tree of the subgraph $G\left[X_{k} \cup Y_{k}\right]$ of $G$, induced by the set $X_{k} \cup Y_{k}$, which is not possible since $G\left[X_{k} \cup Y_{k}\right]$ is not connected. On the other hand, if $u$ and $v$ are non-adjacent vertices of $G_{k}$, without loss of generality we may assume $u \in X_{k}$ and $v \in Y_{k}$. Therefore

$$
d_{G_{k}}(u)+d_{G_{k}}(v)=2((k-1)+(2 k+2))=6 k+2=\frac{3 n-2}{2}
$$

where $n=4 k+2$ is the number of vertices of $G_{k}$. This shows that the degree-sum condition in Theorem 2 is tight. 
Whenever possible we follow the notation of J. A. Bondy and U. S. R. Murty [1].

\section{Proof of Theorem 2}

Suppose the result is false. Then for certain integer $n \geq 4$ and certain arboreal sequence $\sigma=d_{1}, d_{2}, \ldots, d_{n}$ with $1 \leq d_{i} \leq 3$ for $i=1,2, \ldots, n$ there exists a counterexample. That is a labelled graph $G$ with vertex set $V(G)=\left\{w_{1}, w_{2}, \ldots, w_{n}\right\}$ such that $G$ contains no spanning tree with degree sequence $\sigma$ while $d(u)+d(v) \geq \frac{3 n-1}{2}$ for each pair $u, v$ of non-adjacent vertices of $G$. We choose $G$ with the maximum possible number of edges while remaining a counter example with $n$ vertices.

Since $\sigma$ is an arboreal sequence of order $n$, a counterexample cannot be a complete graph of order $n$. Let $u, v$ be non-adjacent vertices of $G$. By the choice of $G$, the graph $G+u v$ is not a counterexample and contains a spanning tree $T$ with degree sequence $\sigma$. Therefore $G$ contains a spanning forest $F=T-u v$ with exactly two components $T_{u}$ and $T_{v}$ with $u \in V\left(T_{u}\right)$ and $v \in V\left(T_{v}\right)$ such that $d_{F}(u)=d_{i}-1, d_{F}(v)=d_{j}-1$ and $d_{f}\left(w_{r}\right)=d_{r}$ for each vertex $u \neq w_{r} \neq v$, where $i$ and $j$ are such that $u=w_{i}$ and $v=w_{j}$.

Orient the edges of $F$ in such a way that $T_{u}$ and $T_{v}$ become outdirected trees $\vec{T}_{u}$ and $\vec{T}_{v}$ with roots $u$ and $v$, respectively. For each vertex $u \neq w \neq v$ let $w^{-}$denote the unique vertex of $G$ such that the edge $w^{-} w$ is oriented from $w^{-}$to $w$ in $\vec{F}$. Let

$$
\begin{gathered}
A_{u}=\left\{y^{-} \in V\left(T_{u}\right): u y \in E(G)\right\}, B_{u}=\left\{x \in V\left(T_{u}\right): v x \in E(G)\right\}, \\
A_{v}=\left\{y^{-} \in V\left(T_{v}\right): v y \in E(G)\right\} \text { and } B_{v}=\left\{x \in V\left(T_{v}\right): u x \in E(G)\right\} .
\end{gathered}
$$

Notice that $\left|A_{u} \cap B_{u}\right|=0$, for if $z^{-} \in A_{u} \cap B_{u}$, then $\left(F-z^{-} z\right)+\left\{u z, v z^{-}\right\}$would be a spanning tree of $G$ with degree sequence $\sigma$, which is not possible (See Fig. 2). Analogously $\left|A_{v} \cap B_{v}\right|=0$ and therefore

$$
\left|A_{u}\right|+\left|B_{u}\right|=\left|A_{u} \cup B_{u}\right| \leq n_{u} \text { and }\left|A_{v}\right|+\left|B_{v}\right|=\left|A_{v} \cup B_{v}\right| \leq n_{v},
$$

where $n_{u}$ and $n_{v}$ are the number of vertices of $T_{u}$ and $T_{v}$, respectively.

In an abuse of notation, for each vertex $x$ of $G$ we denote by $d_{u}(x)$ and $d_{v}(x)$ the number of vertices of $T_{u}$ and $T_{v}$, respectively, which are adjacent to $x$ in $G$. Clearly

$$
\left|B_{u}\right|=d_{u}(v) \text { and }\left|B_{v}\right|=d_{v}(u) .
$$

Also notice that

$$
\left|A_{u}\right| \geq \frac{d_{u}(u)}{2} \text { and }\left|A_{v}\right| \geq \frac{d_{v}(v)}{2}
$$

since the out-degree of each vertex of $\vec{F}$ is at most 2. Then

$$
\frac{d_{u}(u)}{2}+d_{u}(v) \leq\left|A_{u}\right|+\left|B_{u}\right| \leq n_{u} \text { and } \frac{d_{v}(v)}{2}+d_{v}(u) \leq\left|A_{v}\right|+\left|B_{v}\right| \leq n_{v} .
$$



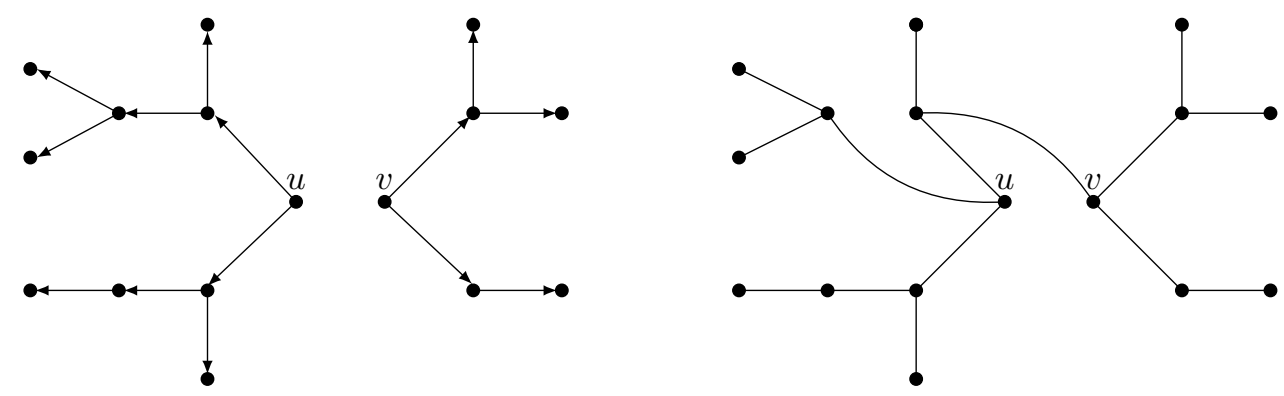

FIGURE 2. Forest $F$ with $z^{-} \in A_{u} \cap B_{u}$ (left). Tree $\left(F-z^{-} z\right)+\left\{u z, v z^{-}\right\}$(right).

Therefore

$$
d_{u}(u)+2 d_{u}(v) \leq 2 n_{u} \text { and } d_{v}(v)+2 d_{v}(u) \leq 2 n_{v}
$$

Since $u$ is not adjacent to $u$ and $v$ is not adjacent to $v$ in $G, d_{u}(u) \leq n_{u}-1$ and $d_{v}(v) \leq n_{v}-1$. Adding these to the previous inequalities we obtain

$$
2 d_{u}(u)+2 d_{v}(v) \leq 3 n_{u}-1 \text { and } 2 d_{v}(v)+2 d_{v}(u) \leq 3 n_{v}-1 .
$$

These imply

$$
2 d(u)+2 d(v)=\left(2 d_{u}(u)+2 d_{v}(u)\right)+\left(2 d_{v}(v)+2 d_{u}(v)\right) \leq 3\left(n_{u}+n_{v}\right)-2=3 n-2,
$$

which is not possible since $d(u)+d(v) \geq \frac{3 n-1}{2}$.

\section{FINAL REMARKS}

With the same approach, we can prove the following generalization of Theorem 2.

Theorem 3. Let $r \geq 2$ be an integer and $G$ be a labelled graph with vertex set $V(G)=$ $\left\{v_{1}, v_{2}, \ldots, v_{n}\right\}$ with $n \geq r+1$. If $d(u)+d(v) \geq \frac{(2 r-3) n-(2 r-5)}{r-1}$ for each pair $u$, $v$ of nonadjacent vertices, then $G$ has a spanning tree $T$ with degree sequence $\sigma$ for each arboreal sequence $\sigma=d_{1}, d_{2}, \ldots, d_{n}$ with $1 \leq d_{i} \leq r$ for $i=1,2, \ldots, n$.

Let $r \geq 2$ be an integer. For each positive integer $k$ let $X_{k}=\left\{x_{1}, x_{2}, \ldots, x_{k}\right\}, Y_{k}=$ $\left\{y_{1}, y_{2}, \ldots, y_{k}\right\}$ and $Z_{k, r}=\left\{z_{1}, z_{2}, \ldots, z_{2 k(r-2)+2}\right\}$ be pairwise disjoint vertex sets and let $G_{k, r}$ be the complete graph with vertex set $X_{k} \cup Y_{k} \cup Z_{k, r}$ with all edges $x_{i} y_{i}, 1 \leq i, j \leq k$ removed. As for the graphs $G_{k}=G_{k, 3}$ in the introduction, we claim that the graphs $G_{k, r}$ show that the degree-sum condition in Theorem 3 is also tight. 


\section{REFERENCES}

[1] Bondy. J. A.; Murty, U. S. R.: Graph Theory with Applications, The McMillan Press, (1976).

[2] Broersma, H.; Tuinstra, H.: Independence tees and Hamilton cycles, J. Graph Theory. 29 (1998), $227-237$.

[3] Ore, O.: Note on Hamilton Circuits, American Mathematical Monthly. 67 (1960), 55.

[4] Rivera-Campo, E.: Spanning trees with small degrees and few leaves, Applied Mathematics Letters. 25 (2012), $1444-1446$.

[5] Win, S.: Existenz von Gerütsen Mit vorgeschriebenem Maximalgraden in Graphen, Abh. Math. Seminar Univ. Hamburg 43 (1975), 263 - 267. 